\title{
Quotient of Ideals of an Intuitionistic Fuzzy Lattice
}

\author{
K. V. Thomas ${ }^{1}$ and Latha S. Nair ${ }^{2}$ \\ ${ }^{1}$ Bharata Mata College, Mahatma Gandhi University, Kochi-Kerala, Thrikkakara 682 021, India \\ ${ }^{2}$ Mar Athanasius College, Mahatma Gandhi University, Kochi, Kothamangalam, Kerala 686 666, India
}

Correspondence should be addressed to Latha S. Nair, lathavichattu@gmail.com

Received 22 September 2010; Revised 10 November 2010; Accepted 2 December 2010

Academic Editor: José Luis Verdegay

Copyright (@ 2010 K. V. Thomas and L. S. Nair. This is an open access article distributed under the Creative Commons Attribution License, which permits unrestricted use, distribution, and reproduction in any medium, provided the original work is properly cited.

The concept of intuitionistic fuzzy ideal of an intuitionistic fuzzy lattice is introduced, and its certain characterizations are provided. We defined the quotient (or residual) of ideals of an intuitionistic fuzzy sublattice and studied their properties.

\section{Introduction}

The concept of intuitionistic fuzzy sets was introduced by Atanassov $[1,2]$ as a generalization of that of fuzzy sets and it is a very effective tool to study the case of vagueness. Further many researches applied this notion in various branches of mathematics especially in algebra and defined intuitionistic fuzzy subgroups (IFG), intuitionistic fuzzy subrings (IFR), and intuitionistic fuzzy sublattice (IFL), and so forth. In the last five years there are so many articles appeared in this direction. Kim [3], Kim and Jun [4], Kim and Lee [5], introduced different types of IFI's in Semigroups. Torkzadeh and Zahedi [6] defined intuitionistic fuzzy commutative hyper K-ideals, Akram and Dudek [7] defined intuitionistic fuzzy Lie ideals of Lie algebras, and Hur et al. [8] introduced intuitionistic fuzzy prime ideals of a Ring.

The concept of ideal of a fuzzy subring was introduced by Mordeson and Malik in [9]. After that N Ajmal and A.S Prajapathi introduced the concept of residual of ideals of an L-Ring in [10]. Motivated by this, in this paper we first defined the intuitionistic fuzzy ideal of an IFL and certain characterizations are given. Lastly we defined quotients (residuals) of ideals of an intuitionistic fuzzy sublattice and studied their properties.

\section{Preliminaries}

We recall the following definitions and results which will be used in the sequel. Throughout this paper $L$ stands for a lattice $(L, \vee, \wedge)$ with zero element " 0 " and unit element " 1 ".
Definition 1 (see [1]). Let $X$ be a nonempty set. An intuitionistic fuzzy set [IFS] $A$ of $X$ is an object of the following form $A=\left\{\left\langle x, \mu_{A}(x), v_{A}(x)\right\rangle \mid x \in X\right\}$, where $\mu_{\mathrm{A}}: X \rightarrow[01]$ and $\nu_{A}: X \rightarrow[01]$ define the degree of membership and the degree of non membership of the element $x \in X$, respectively, and $\forall x \in X, 0 \leq \mu_{A}(x)+\nu_{A}(x) \leq 1$.

The set of all IFS's on $X$ is denoted by IFS $(X)$.

Definition 2 (see [1]). If $A=\left\{\left\langle x, \mu_{A}(x), \nu_{A}(x)\right\rangle \mid x \in S\right\}$ and $B=\left\{\left\langle x, \mu_{B}(x), \nu_{B}(x)\right\rangle \mid x \in S\right\}$ are any two IFS of $X$ then

(i) $A \subseteq B \Leftrightarrow \mu_{A}(x) \leq \mu_{B}$ and $\nu_{A}(x) \geq \nu_{B}(x) \forall x \in X$;

(ii) $A=B \Leftrightarrow \mu_{A}(x)=\mu_{B}(x)$ and $\nu_{A}(x)=\nu_{B}(x)$;

(iii) $\bar{A}=\left\{\left\langle x, v_{A}(x), \mu_{A}(x)\right\rangle \mid x \in X\right\}$;

(iv) $[A]=\left\{\left\langle x, \mu_{A}(x), \mu_{A}^{c}(x)\right\rangle \mid x \in X\right\}$, where $\mu_{A}^{c}(x)=$ $1-\mu_{A}(x)$

(v) $\langle A\rangle=\left\{\left\langle x, v_{A}^{c}(x), v_{A}(x)\right\rangle \mid x \in X\right\}$, where $\nu_{A}^{c}(x)=$ $1-\nu_{A}(x)$

(vi) $A \cap B=\left\{\left\langle x, \min \left\{\mu_{A}(x), \mu_{B}(x)\right\}, \max \left\{\nu_{A}(x), \nu_{B}(x)\right\}\right\rangle \mid\right.$ $x \in X\}=\left\{\left\langle x, \mu_{A \cap B}(x), \nu_{A \cap B}(x)\right\rangle \mid x \in X\right\} ;$

(vii) $A \cup B=\left\{\left\langle x, \max \left\{\mu_{A}(x), \mu_{B}(x)\right\}, \min \left\{v_{A}(x), \nu_{B}(x)\right\}\right\rangle \mid\right.$ $x \in X\}=\left\{\left\langle x, \mu_{A \cup B}(x), \nu_{A \cup B}(x)\right\rangle \mid x \in X\right\}$.

Definition 3 (see [11]). Let $L$ be a lattice and $A=$ $\left\{\left\langle x, \mu_{A}(x), \nu_{A}(x)\right\rangle \mid x \in L\right\}$ be an IFS of $L$. Then $A$ is called an intuitionistic fuzzy sublattice [IFL] of $L$ if the following conditions are satisfied. 
(i) $\mu_{A}(x \vee y) \geq \min \left\{\mu_{A}(x), \mu_{A}(y)\right\}$;

(ii) $\mu_{A}(x \wedge y) \geq \min \left\{\mu_{A}(x), \mu_{A}(y)\right\}$;

(iii) $\nu_{A}(x \vee y) \leq \max \left\{\nu_{A}(x), \nu_{A}(y)\right\}$;

(iv) $v_{A}(x \wedge y) \leq \max \left\{v_{A}(x), v_{A}(y)\right\}, \forall x, y \in L$.

The set of all intuitionist fuzzy sublattices (IFL's) of $L$ is denoted as IFL $(L)$.

Definition 4 (see [11]). An IFS $A$ of $L$ is called an intuitionistic fuzzy ideal (IFI) of $L$ if the following conditions are satisfied.

(i) $\mu_{A}(x \vee y) \geq \min \left\{\mu_{A}(x), \mu_{A}(y)\right\}$;

(ii) $\mu_{A}(x \wedge y) \geq \max \left\{\mu_{A}(x), \mu_{A}(y)\right\}$;

(iii) $\nu_{A}(x \vee y) \leq \max \left\{\nu_{A}(x), v_{A}(y)\right\}$;

(iv) $v_{A}(x \wedge y) \leq \min \left\{v_{A}(x), v_{A}(y)\right\}, \forall x, y \in L$.

The set of all IFI's of $L$ is denoted as IFI $(L)$.

Definition 5 (see [12]). Let $A, B \in \operatorname{IFS}(L)$. Then we define an IFS $(L)$,

(i) $A+B=\left\{\left\langle z, \mu_{A+B}(z), v_{A+B}(z)\right\rangle \mid z \in L\right\}$, where

$$
\begin{aligned}
& \mu_{A+B}(z)=\sup _{z=x \vee y}\left\{\min \left\{\mu_{A}(x), \mu_{B}(y)\right\}\right\}, \\
& \nu_{A+B}(z)=\inf _{z=x \vee y}\left\{\max \left\{\nu_{A}(x), \nu_{B}(y)\right\}\right\} .
\end{aligned}
$$

(ii) $A B=\left\{\left\langle z, \mu_{A B}(z), v_{A B}(z)\right\rangle \mid z \in L\right\}$, where

$$
\begin{aligned}
& \mu_{A B}(z)=\sup _{z=x \wedge y}\left\{\min \left\{\mu_{A}(x), \mu_{B}(y)\right\}\right\} \\
& \nu_{A B}(z)=\inf _{z=x \wedge y}\left\{\max \left\{\nu_{A}(x), \nu_{B}(y)\right\}\right\} .
\end{aligned}
$$

(iii) $A \cdot B=\left\{\left\langle z, \mu_{A \cdot B}(z), v_{A \cdot B}(z)\right\rangle \mid z \in L\right\}$ where

$$
\begin{aligned}
& \mu_{A \cdot B}(z)=\sup _{z=\vee_{i=1}^{n}\left(x_{i} \wedge y_{i}\right)}\left(\min _{i}\left\{\min \left\{\mu_{A}\left(x_{i}\right), \mu_{B}\left(y_{i}\right)\right\}\right\}\right), \\
& v_{A \cdot B}(z)=\inf _{z=\vee_{i=1}^{n}\left(x_{i} \wedge y_{i}\right)}\left(\max _{i}\left\{\max \left\{\nu_{A}\left(x_{i}\right), \nu_{B}\left(y_{i}\right)\right\}\right\}\right)
\end{aligned}
$$

Lemma 1 (see [13]). Let $A, B$, and $C$ be IFS (L), then the following assertions hold.

(1) $A B=B A, A+B=B+A, A \cdot B=B \cdot A$.

(2) $A B \subseteq A \cdot B$.

(3) $C(A+B) \subseteq C A+C B$.

(4) $(C+B) A \subseteq C A+B A$.

(5) $(A \cap B) C \subseteq A C \cap B C$.

(6) $A \subseteq B \Rightarrow A C \subseteq B C$ and $A \cdot C \subseteq B \cdot C$.

Lemma 2 (see [13]). Let $A$ be an IFL of L, then

(1) $A+A=A$.

(2) $A A=A$.

\section{Ideal of an Intuitionistic Fuzzy Lattice}

In this section we define the ideal of an IFL, and give some characterization of these ideals in terms of operations on IFS $(L)$. We also used $\vee$ and $\wedge$ to represent maximum and minimum, respectively, which is clear from the context.

Definition 6. Let $A$ be an IFL of $L$ and $B$ an IFS of $L$ with $B \subseteq A$. Then $B$ is called an intuitionistic fuzzy ideal (IFI) of $A$ if the following conditions are satisfied.

(i) $\mu_{B}(x \vee y) \geq \mu_{B}(x) \wedge \mu_{B}(y)$.

(ii) $\mu_{B}(x \wedge y) \geq\left[\mu_{A}(x) \wedge \mu_{B}(y)\right] \vee\left[\mu_{B}(x) \wedge \mu_{A}(y)\right]$.

(iii) $\nu_{B}(x \vee y) \leq \nu_{B}(x) \vee v_{B}(y)$.

(iv) $v_{B}(x \wedge y) \leq\left[v_{A}(x) \vee v_{B}(y)\right] \wedge\left[\nu_{B}(x) \vee v_{A}(y)\right] \forall x, y \in$ $L$.

If $B$ IFI of $A$, then we write $B \triangleleft A$

Example 1. Consider the lattice $L=\{1,2,5,10\}$ under divisibility.

Let $A=\left\{\left\langle x, \mu_{A}(x), v_{A}(x)\right\rangle \mid x \in L\right\}$ be an IFL of $L$ defined by $\langle 1, .5, .1\rangle,\langle 2, .4, .5\rangle,\langle 5, .4, .3\rangle,\langle 10, .7, .3\rangle$ and $B=\left\{\left\langle x, \mu_{B}(x), \nu_{B}(x)\right\rangle \mid x \in L\right\}$ be an IFS of $\mathrm{L}$ given by $\langle 1, .5, .3\rangle,\langle 2, .4, .5\rangle,\langle 5, .3, .4\rangle,\langle 10, .3, .4\rangle$. Clearly $B \triangleleft A$.

Definition 7. Let $A$ be an IFL and $B$ is also an IFL with $B \subseteq A$. Then $B$ is called an intuitionistic fuzzy sublattice of $A$.

Lemma 3. The intersection of two IFI's of $A$ is again an IFI of A.

Proof. Let $B, C$ be IFI's of $A$. Then we can prove that $B \cap C$ is also an IFI of $A$. Since $B \subseteq A$ and $C \subseteq A$, we have $B \cap C \subseteq A$ Also

$$
\begin{aligned}
\mu_{B \cap C}(x \vee y)= & \min \left\{\mu_{B}(x \vee y), \mu_{C}(x \vee y)\right\} \\
\geq & \min \left\{\mu_{B}(x) \wedge \mu_{B}(y), \mu_{C}(x) \wedge \mu_{C}(y)\right\}, \\
& \text { since } B \text { and } C \text { are IFI's of } A . \\
\geq & \min \left\{\mu_{B}(x) \wedge \mu_{C}(x), \mu_{B}(y) \wedge \mu_{C}(y)\right\} \\
\geq & \min \left\{\mu_{B \cap C}(x), \mu_{B \cap C}(y)\right\} \\
\geq & \mu_{B \cap C}(x) \wedge \mu_{B \cap C}(y), \\
\mu_{B \cap C}(x \wedge y)= & \min \left\{\mu_{B}(x \wedge y), \mu_{C}(x \wedge y)\right\} \\
\geq & \min \left\{\left[\mu_{B}(x) \wedge \mu_{A}(y)\right] \vee\left[\mu_{B}(y) \wedge \mu_{A}(x)\right],\right. \\
& \left.\quad\left[\mu_{C}(x) \wedge \mu_{A}(y)\right] \vee\left[\mu_{A}(x) \wedge \mu_{C}(y)\right]\right\} \\
& \quad \operatorname{since} B \text { and } C \text { are IFI's of } A . \\
\geq & \min \left\{\mu_{B}(x), \mu_{C}(x)\right\} \wedge \mu_{A}(y) \\
& \vee \min \left\{\mu_{B}(y), \mu_{C}(y)\right\} \wedge \mu_{A}(x) \\
\geq & {\left[\mu_{B \cap C}(x) \wedge \mu_{A}(y)\right] \vee\left[\mu_{B \cap C}(y) \wedge \mu_{A}(x)\right] . }
\end{aligned}
$$


Also

$$
\begin{aligned}
& \nu_{B \cap C}(x \vee y)=\max \left\{\nu_{B}(x \vee y), \nu_{C}(x \vee y)\right\} \\
& \leq \max \left\{\nu_{B}(x) \vee \nu_{B}(y), \nu_{C}(x) \vee \nu_{C}(y)\right\}, \\
& \text { since } B \text { and } C \text { are IFI's of } A \text {. } \\
& \leq \max \left\{v_{B}(x) \vee v_{C}(x), v_{B}(y) \vee v_{C}(y)\right\} \\
& \leq \max \left\{v_{B \cap C}(x), v_{B \cap C}(y)\right\} \\
& \leq v_{B \cap C}(x) \vee v_{B \cap C}(y), \\
& \underset{B \cap C}{\nu}(x \wedge y)=\max \left\{\nu_{B}(x \wedge y), \nu_{C}(x \wedge y)\right\} \\
& \leq \max \left\{\left[\nu_{B}(x) \vee v_{A}(y)\right] \wedge\left[\nu_{B}(y) \vee v_{A}(x)\right],\right. \\
& \left.\left[\nu_{C}(x) \vee v_{A}(y)\right] \wedge\left[\nu_{A}(x) \vee v_{C}(y)\right]\right\}, \\
& \text { since } B \text { and } C \text { are IFI's of } A \text {. } \\
& \leq \max \left\{\nu_{B}(x), \nu_{C}(x)\right\} \vee \nu_{A}(y) \\
& \wedge \max \left\{v_{B}(y), v_{C}(y)\right\} \vee v_{A}(x) \\
& \leq\left[\nu_{B \cap C}(x) \vee \nu_{A}(y)\right] \wedge\left[\nu_{B \cap C}(y) \vee \nu_{A}(x)\right] .
\end{aligned}
$$

Hence, $B \cap C$ is an IFI of $A$.

Theorem 1. Let $A$ an IFL and $B$ an IFS of $L$ with $B \subseteq A$. Then $B$ is an IFI of $A$ if and only if

$$
\begin{aligned}
& \text { (1) } \mu_{B}(x \vee y) \geq \mu_{B}(x) \wedge \mu_{B}(y), \\
& \text { (2) } \nu_{B}(x \vee y) \leq \nu_{B}(x) \vee v_{B}(y), \\
& \text { (3) } A B \subseteq B .
\end{aligned}
$$

Proof. Suppose that conditions (1), (2), and (3) hold. Then we prove that $B$ is an IFI of $A$.

We have

$$
\begin{aligned}
\mu_{B}(x \wedge y) & \geq \mu_{A B}(x \wedge y), \quad \text { since } B \supseteq A B \\
& =\sup _{x \wedge y=x_{i} \wedge y_{i}}\left(\mu_{A}\left(x_{i}\right) \wedge \mu_{B}\left(y_{i}\right)\right) \\
& \geq \mu_{A}(x) \wedge \mu_{B}(y) .
\end{aligned}
$$

Similarly

$$
\mu_{B}(x \wedge y) \geq \mu_{B}(x) \wedge \mu_{A}(y) \text {, since } B \supseteq A B=B A \text {. }
$$

Hence

$$
\mu_{B}(x \wedge y) \geq\left[\mu_{A}(x) \wedge \mu_{B}(y)\right] \vee\left[\mu_{B}(x) \wedge \mu_{A}(y)\right] .
$$

Also

$$
\begin{aligned}
\nu_{B}(x \wedge y) & \leq \nu_{A B}(x \wedge y), \quad \text { since } B \supseteq A B \\
& =\inf _{x \wedge y=x_{i} \wedge y_{i}}\left(\nu_{A}\left(x_{i}\right) \vee \nu_{B}\left(y_{i}\right)\right) \\
& \leq \nu_{A}(x) \vee \nu_{B}(y) .
\end{aligned}
$$

Similarly

$$
\nu_{B}(x \wedge y) \leq \nu_{B}(x) \vee \nu_{A}(y) \text {, since } B \supseteq A B=B A .
$$

Hence

$$
\nu_{B}(x \wedge y) \leq\left[\nu_{A}(x) \vee \nu_{B}(y)\right] \wedge\left[\nu_{B}(x) \vee \nu_{A}(y)\right] .
$$

So from (1), (2), (a), and (b) $B$ is an IFI of $A$.

Conversely suppose $B$ is an IFI of $A$. Then obviously conditions (1) and (2) holds. Also we have

$$
\begin{aligned}
& \mu_{B}(x \wedge y) \geq \mu_{A}(x) \wedge \mu_{B}(y), \\
& \nu_{B}(x \wedge y) \leq \nu_{A}(x) \vee \nu_{B}(y), \quad \forall x, y \in L .
\end{aligned}
$$

So $\forall z \in L$ with $z=x \wedge y$

$$
\begin{aligned}
& \mu_{B}(z) \geq \bigvee_{z=x \wedge y}\left[\mu_{A}(x) \wedge \mu_{B}(y)\right]=\mu_{A B}(z), \\
& \nu_{B}(z) \leq \bigwedge_{z=x \wedge y}\left[v_{A}(x) \vee v_{B}(y)\right]=v_{A B}(z) .
\end{aligned}
$$

Hence $A B \subseteq B$.

Theorem 2. Let $A$ be an IFL of $L$ and $B$ an IFS with $B \subseteq A$. Then $B$ is an IFI of $A$ if and only if

(1) $\mu_{B}(x \vee y) \geq \mu_{B}(x) \wedge \mu_{B}(y)$,

(2) $\nu_{B}(x \vee y) \leq \nu_{B}(x) \vee v_{B}(y)$

(3) $A \cdot B \subseteq B$.

Proof. Suppose conditions (1), (2), and (3) holds. We prove $B$ is an IFI of $A$.

We have

$$
\begin{aligned}
\mu_{B}(x \wedge y) & \geq \mu_{A \cdot B}(x \wedge y), \quad \text { since } B \supseteq A \cdot B \\
& =\sup _{x \wedge y=v_{i=1}^{n}\left(x_{i} \wedge y_{i}\right)}\left[\bigwedge_{i=1}^{n}\left(\mu_{A}\left(x_{i}\right) \wedge \mu_{B}\left(y_{i}\right)\right)\right] \\
& \geq \mu_{A}(x) \wedge \mu_{B}(y) .
\end{aligned}
$$

Similarly, we can obtain

$$
\mu_{B}(x \wedge y) \geq \mu_{B}(x) \wedge \mu_{A}(y) \text {, since } B \supseteq A \cdot B=B \cdot A \text {. }
$$

Hence

$$
\mu_{B}(x \wedge y) \geq\left[\mu_{A}(x) \wedge \mu_{B}(y)\right] \vee\left[\mu_{B}(x) \wedge \mu_{A}(y)\right] .
$$

Also

$$
\begin{aligned}
\nu_{B}(x \wedge y) & \leq \nu_{A \cdot B}(x \wedge y), \quad \text { since } B \supseteq A \cdot B \\
& =\inf _{x \wedge y=\bigvee_{i=1}^{n}\left(x_{i \wedge} y_{i}\right)}\left[\bigvee_{i=1}^{n}\left(\nu_{A}\left(x_{i}\right) \vee v_{B}\left(y_{i}\right)\right)\right] \\
& \leq v_{A}(x) \vee v_{B}(y) .
\end{aligned}
$$


Similarly

$$
\nu_{B}(x \wedge y) \leq \nu_{B}(x) \vee v_{A}(y), \quad \text { since } B \supseteq A \cdot B=B \cdot A \text {. }
$$

Hence

$$
v_{B}(x \wedge y) \leq\left[v_{A}(x) \vee v_{B}(y)\right] \wedge\left[\nu_{B}(x) \vee v_{A}(y)\right]
$$

So from (1), (2), (a.1), and (b.1) $B$ is an IFI of $A$.

Conversely suppose that $B$ is an IFI of $A$. Then obviously conditions (1) and (2) hold.

Let $z \in L$ and $z=\bigvee_{i=1}^{n}\left(x_{i} \wedge y_{i}\right)$, where $x_{i} \in A, y_{i} \in B$.

We have

$$
\begin{aligned}
\mu_{B}(z) & =\mu_{B}\left[\bigvee_{i=1}^{n}\left(x_{i} \wedge y_{i}\right)\right] \geq \bigwedge_{i=1}^{n} \mu_{B}\left(x_{i} \wedge y_{i}\right) \\
& \geq \bigwedge_{i=1}^{n}\left\{\mu_{A}\left(x_{i}\right) \wedge \mu_{B}\left(y_{i}\right)\right\}, \quad \text { since } B \text { IFI of } A .
\end{aligned}
$$

Thus

$$
\mu_{B}(z) \geq \bigvee\left[\bigwedge_{i=1}^{n}\left\{\mu_{A}\left(x_{i}\right) \wedge \mu_{B}\left(y_{i}\right)\right\}\right]=\mu_{A \cdot B}(z) .
$$

Also

$$
\begin{aligned}
\nu_{B}(z) & =v_{B}\left[\bigvee_{i=1}^{n}\left(x_{i} \wedge y_{i}\right)\right] \leq \bigvee_{i=1}^{n} v_{B}\left(x_{i} \wedge y_{i}\right) \\
& \leq \bigvee_{i=1}^{n}\left\{v_{A}\left(x_{i}\right) \vee v_{B}\left(y_{i}\right)\right\}, \quad \text { since } B \text { IFI of } A .
\end{aligned}
$$

Thus

$$
v_{B}(z) \leq \wedge\left[\bigvee_{i=1}^{n}\left\{v_{A}\left(x_{i}\right) \vee v_{B}\left(y_{i}\right)\right\}\right]=v_{A \cdot B}(z)
$$

Hence $A \cdot B \subseteq B$.

Theorem 3. Let $A$ be an IFL of $L$ and $B, C$ are IFI's of $A$. Then $B+C$ is an IFI of $A$.

Proof. We have

$$
\begin{aligned}
& \mu_{B+C}(x \vee y) \geq \mu_{B+C}(x) \wedge \mu_{B+C}(y), \\
& v_{B+C}(x \vee y) \leq v_{B+C}(x) \vee v_{B+C}(y)
\end{aligned}
$$

(by [11, Theorem 5.2]).

And

$$
\begin{aligned}
& A(B+C) \subseteq A B+A C \subseteq B+C, \\
& (B+C) A \subseteq B A+C A \subseteq B+C .
\end{aligned}
$$

(by Lemma 1 and Theorem 1 ).

Hence $B+C$ is an IFI of $A$.

\section{Quotient of Ideals}

Here first we define the residual of ideals of an IFL and prove that the residual of ideals is again an IFI of the IFL. Moreover we establish that it is the largest ideal with respect to some property on the operation $\cdot$.

Definition 8. Let $A$ be an IFL of $L$ and $B, C$ be IFI's of $A$. Then the quotient (residual) of $B$ by $C$ denoted as $B / C$ is defined by

$$
B / C=\cup\{D / D \triangleleft A, D C \subseteq B\} .
$$

Theorem 4. Let $A$ be an IFL of $L$ and $B, C$ are IFI's of $A$. Then the quotient $B / C$ is an IFI of $A$. Also $B \subseteq B / C \subseteq A$.

Proof. Let $\eta=\{D / D \triangleleft A, D C \subseteq B\}$. Suppose $D, D^{\prime} \in$ $\eta$. Then $D$ and $D^{\prime}$ are IFI's of $A$ such that $D C \subseteq B$ and $D^{\prime} C \subseteq B$. Then by Theorem $3 D+D^{\prime}$ is an IFI of $A$. So by Lemmas 1 and $2\left(D+D^{\prime}\right) C \subseteq D C+D^{\prime} C \subseteq B+B=B$. Thus $D+D^{\prime} \in \eta$. Now

$$
\begin{aligned}
\mu_{B / C}(x) \wedge \mu_{B / C}(y) & =\left[\bigvee_{D \in \eta} \mu_{D}(x)\right] \wedge\left[\bigvee_{D^{\prime} \in \eta} \mu_{D^{\prime}}(y)\right] \\
& =\bigvee\left\{\mu_{D}(x) \wedge \mu_{D^{\prime}}(y) / D, D^{\prime} \in \eta\right\} \\
& \leq \bigvee\left\{\mu_{D+D^{\prime}}(x \vee y) / D, D^{\prime} \in \eta\right\} \\
& \leq \mu_{B / C}(x \vee y), \quad \text { since } D+D^{\prime} \in \eta .
\end{aligned}
$$

That is,

$$
\mu_{B / C}(x \vee y) \geq \mu_{B / C}(x) \wedge \mu_{B / C}(y)
$$

Also

$$
\begin{aligned}
\mu_{B / C}(x \wedge y) & =\bigvee_{D \in \eta} \mu_{D}(x \wedge y) \\
& \geq \bigvee_{D \in \eta}\left\{\mu_{D}(x) \wedge \mu_{A}(y)\right\}, \quad \text { since } D \triangleleft A \\
& =\left[\bigvee_{D \in \eta} \mu_{D}(x)\right] \wedge \mu_{A}(y) \\
& =\mu_{B / C}(x) \wedge \mu_{A}(y) .
\end{aligned}
$$

Similarly

$$
\mu_{B / C}(x \wedge y) \geq \mu_{B / C}(y) \wedge \mu_{A}(x) .
$$

Thus

$$
\mu_{B / C}(x \wedge y) \geq\left[\mu_{B / C}(x) \wedge \mu_{A}(y)\right] \vee\left[\mu_{B / C}(y) \wedge \mu_{A}(x)\right] .
$$


Now

$$
\begin{aligned}
v_{B / C}(x) \vee v_{B / C}(y) & =\left[\bigwedge_{D \in \eta} v_{D}(x)\right] \vee\left[\bigwedge_{D^{\prime} \in \eta} v_{D^{\prime}}(y)\right] \\
& =\bigwedge\left\{v_{D}(x) \vee v_{D^{\prime}}(y) / D, D^{\prime} \in \eta\right\} \\
& \geq \bigwedge\left\{v_{D+D^{\prime}}(x \vee y) / D, D^{\prime} \in \eta\right\} \\
& \geq v_{B / C}(x \vee y), \quad \text { since } D+D^{\prime} \in \eta .
\end{aligned}
$$

That is

$$
v_{B / C}(x \vee y) \leq v_{B / C}(x) \vee v_{B / C}(y)
$$

Also

$$
\begin{aligned}
\nu_{B / C}(x \wedge y) & =\bigwedge_{D \in \eta} v_{D}(x \wedge y) \\
& \leq \bigwedge_{D \in \eta}\left\{v_{D}(x) \vee v_{A}(y)\right\}, \quad \text { since } D \triangleleft A \\
& =\left[\bigwedge_{D \in \eta} v(x)\right] \vee v_{A}(y) \\
& =v_{B / C}(x) \vee v_{A}(y) .
\end{aligned}
$$

Similarly

$$
v_{B / C}(x \wedge y) \leq v_{B / C}(y) \vee v_{A}(x) .
$$

Thus

$$
\nu_{B / C}(x \wedge y) \leq\left[\nu_{B / C}(x) \vee v_{A}(y)\right] \wedge\left[\nu_{B / C}(y) \vee \nu_{A}(x)\right] .
$$

From (24), (27), (29), and (32) $B / C$ is an IFI of $A$.

Clearly $B / C \subseteq A$.

Since $B$ is an IFI of $A, B A \subseteq B$ (by Theorem 1).

Since $C \subseteq A$, by Lemma $1 B C \subseteq B A \subseteq B$. Hence $B \in \eta$. So $B \subseteq B / C$.

Thus we have

$$
B \subseteq B / C \subseteq A .
$$

Theorem 5. Let $A$ be an IFL and $B, C$ be IFI's of $A$. Then $B / C$ is the largest IFI of $A$ with the property $(B / C) \cdot C \subseteq B$.

Proof. Let $\eta=\{D / D \triangleleft A$ and $D C \subseteq B\}$. We have $B / C=$ $\bigcup_{D \in \eta} D$ Let $x \in L$ such that $x=\bigvee_{i=1}^{n}\left(a_{i} \wedge b_{i}\right)$.

Then

$$
\mu_{B}\left(a_{i} \wedge b_{i}\right) \geq \mu_{D C}\left(a_{i} \wedge b_{i}\right) \geq \mu_{D}\left(a_{i}\right) \wedge \mu_{C}\left(b_{i}\right), \quad \forall D \in \eta .
$$

So

$$
\begin{aligned}
\mu_{B}\left(a_{i} \wedge b_{i}\right) & \geq \bigvee_{D \in \eta}\left[\mu_{D}\left(a_{i}\right) \wedge \mu_{C}\left(b_{i}\right)\right] \\
& =\left[\bigvee_{D \in \eta} \mu_{D}\left(a_{i}\right)\right] \wedge \mu_{C}\left(b_{i}\right) \\
& =\mu_{B / C}\left(a_{i}\right) \wedge \mu_{C}\left(b_{i}\right) .
\end{aligned}
$$

Hence

$$
\begin{aligned}
\mu_{B}(x) & =\mu_{B}\left(\bigvee_{i=1}^{n}\left(a_{i} \wedge b_{i}\right)\right) \geq \bigwedge_{i=1}^{n} \mu_{B}\left(a_{i} \wedge b_{i}\right), \\
& \text { since } B \text { is an IFI of } A \\
& \geq \bigwedge_{i=1}^{n}\left[\mu_{B / C}\left(a_{i}\right) \wedge \mu_{C}\left(b_{i}\right)\right] .
\end{aligned}
$$

Consequently

$$
\begin{aligned}
\mu_{B}(x) & \geq \bigvee\left\{\bigwedge_{i=1}^{n}\left[\mu_{B / C}\left(a_{i}\right) \wedge \mu_{C}\left(b_{i}\right)\right] / x=\bigvee_{i=1}^{n}\left(a_{i} \wedge b_{i}\right)\right\} \\
& =\mu_{(B / C) \cdot C}(x) .
\end{aligned}
$$

Also

$$
\nu_{B}\left(a_{i} \wedge b_{i}\right) \leq \nu_{D C}\left(a_{i} \wedge b_{i}\right) \leq \nu_{D}\left(a_{i}\right) \vee v_{C}\left(b_{i}\right), \quad \forall D \in \eta .
$$

So

$$
\begin{aligned}
\nu_{B}\left(a_{i} \wedge b_{i}\right) & \leq \bigwedge_{D \in \eta}\left[\nu_{D}\left(a_{i}\right) \vee v_{C}\left(b_{i}\right)\right] \\
& =\left[\bigwedge_{D \in \eta} v_{D}\left(a_{i}\right)\right] \vee v_{C}\left(b_{i}\right) \\
& =v_{B / C}\left(a_{i}\right) \wedge v_{C}\left(b_{i}\right) .
\end{aligned}
$$

Hence

$$
\nu_{B}(x)=\nu_{B}\left(\bigvee_{i=1}^{n}\left(a_{i} \wedge b_{i}\right)\right) \leq \bigvee_{i=1}^{n} \nu_{B}\left(a_{i} \wedge b_{i}\right)
$$

since $B$ is an IFI of $A$

$$
\leq \bigvee_{i=1}^{n}\left[v_{B / C}\left(a_{i}\right) \wedge v_{C}\left(b_{i}\right)\right]
$$

Consequently

$$
\begin{aligned}
\nu_{B}(x) & \leq \bigwedge\left\{\bigvee_{i=1}^{n}\left[v_{B / C}\left(a_{i}\right) \wedge v_{C}\left(b_{i}\right)\right] / x=\bigvee_{i=1}^{n}\left(a_{i} \wedge b_{i}\right)\right\} \\
& =v_{(B / C) \cdot C}(x) .
\end{aligned}
$$

Thus from (37) and (41) $(B / C) \cdot C \subseteq B$.

If $D$ is an ideal of $A$ such that $D \cdot C \subseteq B$ then $D C \subseteq$ $D \cdot C \subseteq B$. So $D \in \eta$. Hence $D \subseteq B / C$. Thus $B / C$ is the largest IFI of $A$ such that $(B / C) \cdot C \subseteq B$.

Theorem 6. Let $A$ be an IFL and B, C, D be IFI's of $A$. Then the following holds.

(1) If $B \subseteq C$ then $B / D \subseteq C / D$ and $D / C \subseteq D / B$.

(2) If $B \subseteq C$ then $C / B=A$.

(3) $B / B=A$. 
Proof. (1) Let $B \subseteq C$. Write $\eta=\{E / E \triangleleft A$ and $E D \subseteq B\}$ and $\xi=\{E / E \triangleleft A$ and $E D \subseteq C\}$. If $E \in \eta$ then $E \triangleleft A$ and $E D \subseteq B \subseteq C$. Thus $E \in \xi$ and hence $\eta \subseteq \xi$. So $B / D=$ $\bigcup_{E \in \eta} E \subseteq \bigcup_{E \in \xi} E=C / D$.

Similarly, let $\eta_{1}=\{E / E \triangleleft A$ and $E C \subseteq D\}$ and $\xi_{1}=$ $\{E / E \triangleleft A$ and $E B \subseteq D\}$. If $E \in \eta_{1}$ then $E C \subseteq D$. But $B \subseteq C$. So $E B \subseteq E C \subseteq D$. Thus $E \in \xi_{1}$ and hence $\eta_{1} \subseteq \xi_{1}$. So $D / C=$ $\bigcup_{E \in \eta_{1}} E \subseteq \bigcup_{E \in \xi_{1}} E=D / B$.

(2) Let $\eta=\{E / E \triangleleft A$ and $E B \subseteq C\}$. Since $B \triangleleft A$, we have $A B \subseteq B \subseteq C$, and $A \triangleleft A$. Thus $A \in \eta$ and hence $A \subseteq \bigcup_{E \in \eta} E=C / B \subseteq A$, since $C / B$ is an IFI of $A$. Therefore $C / B=A$.

(3) We have $B \subseteq B$. So from (2) $B / B=A$.

Corollary 1. Let $A$ be an IFL of $L$ and B, and C be IFI's of $A$. Then

(1) $(B / C) / B=A$,

(2) $(B / B) / C=A$,

(3) $B /(B \cap C)=A$.

Proof. (1) Since $B \subseteq B / C$, by Theorem 6 (2), $(B / C) / B=A$.

(2) By Theorem 6 (3) $B / B=A$. Since $C \subseteq A=B / B$ by Theorem $6(2),(B / B) / C=A$.

(3) Since $B \triangleleft A$ and $C \triangleleft A$. So $B \cap C \triangleleft A$ and $B \cap C \subseteq B$. Hence by Theorem 6 (2), $B /(B \cap C)=A$.

Theorem 7. Let $A$ be an IFL of $L$ and $B_{i} i=1,2 \ldots \ldots m, C$, are IFI's of $A$. Then

$$
\left(\bigcap_{i=1}^{m} B_{i}\right) / C=\bigcap_{i=1}^{m}\left(B_{i} / C\right) .
$$

Proof. Since $\bigcap_{i=1}^{m} B_{i} \subseteq B_{i}$ by Theorem $6(1)\left(\bigcap_{i=1}^{m} B_{i}\right) / C \subseteq$ $B_{i} / C, \forall i$.

Hence

$$
\left(\bigcap_{i=1}^{m} B_{i}\right) / C \subseteq \bigcap_{i=1}^{m}\left(B_{i} / C\right) .
$$

Let

$$
\begin{aligned}
& \eta_{1}=\left\{E / E \triangleleft A, E C \subseteq B_{1}\right\}, \\
& \eta_{2}=\left\{E / E \triangleleft A, E C \subseteq B_{2}\right\}, \\
& \eta_{3}=\left\{E / E \triangleleft A, E C \subseteq B_{1} \cap B_{2}\right\} .
\end{aligned}
$$

Then $\forall x \in L$

$$
\begin{aligned}
\mu_{B_{1} / C \cap B_{2} / C}(x) & =\mu_{B_{1} / C}(x) \wedge \mu_{B_{2} / C}(x) \\
& =\left(\bigvee_{E \in \eta_{1}} \mu_{E}(x)\right) \wedge\left(\bigvee_{E^{\prime} \in \eta_{2}} \mu_{E^{\prime}}(x)\right) \\
& =\vee\left\{\left[\mu_{E}(x) \wedge \mu_{E^{\prime}}(x)\right] / E \in \eta_{1}, E^{\prime} \in \eta_{2}\right\} .
\end{aligned}
$$

Similarly

$$
\begin{aligned}
\nu_{B_{1} / C \cap B_{2} / C}(x) & =\nu_{B_{1} / C}(x) \vee v_{B_{2} / C}(x) \\
& =\left(\bigwedge_{E \in \eta_{1}} \nu_{E}(x)\right) \vee\left(\bigwedge_{E^{\prime} \in \eta_{2}} v_{E^{\prime}}(x)\right) \\
& =\wedge\left\{\left[\nu_{E}(x) \vee \nu_{E^{\prime}}(x)\right] / E \in \eta_{1}, E^{\prime} \in \eta_{2}\right\} .
\end{aligned}
$$

Now let $E \in \eta_{1}$ and $E^{\prime} \in \eta_{2}$. Then $E C \subseteq B_{1}$ and $E^{\prime} C \subseteq B_{2}$. Also $E \cap E^{\prime}$ IFI of $A$.

So that

$$
\left(E \cap E^{\prime}\right) C \subseteq E C \cap E^{\prime} C \subseteq B_{1} \cap B_{2} .
$$

Thus $E \cap E^{\prime} \in \eta_{3}$. So $\eta_{1} \cap \eta_{2} \subseteq \eta_{3}$.

Hence

$$
\left(B_{1} \cap B_{2}\right) / C=\bigcup_{E \in \eta_{3}} E \supseteq \bigcup_{E \in \eta_{1}, E^{\prime} \in \eta_{2}}\left(E \cap E^{\prime}\right) .
$$

So

$$
\begin{aligned}
\mu_{B_{1} \cap B_{2} / C}(x) & \geq \bigvee \mu_{E \cap E^{\prime}}(x) \\
& =\bigvee\left[\mu_{E}(x) \wedge \mu_{E^{\prime}}(x)\right] \\
& =\mu_{B_{1} / C \cap B_{2} / C}(x), \quad \text { from }(\text { a. } 2), \\
\nu_{B_{1} \cap B_{2} / C}(x) & \leq \bigwedge \nu_{E \cap E^{\prime}}(x) \\
& =\bigwedge\left[\nu_{E}(x) \vee v_{E^{\prime}}(x)\right] \\
& =v_{B_{1} / C \cap B_{2} / C}(x), \quad \text { from (b.2). }
\end{aligned}
$$

Hence

$$
B_{1} \cap B_{2} / C \supseteq B_{1} / C \cap B_{2} / C \text {. }
$$

From (43) and (48) $\left(B_{1} \cap B_{2}\right) / C=B_{1} / C \cap B_{2} / C$. This completes the proof.

Next, we denote the set of all IFI's $\{B i\} i=1,2 \ldots$, $m$ of an IFL $A$ that satisfies the property $\mu_{B_{i}}(0)=\mu_{B j}(0)$ and $\nu_{B_{i}}(0)=\nu_{B j}(0) \forall i, j$ by IFI $\left(A^{*}\right)$. Then we have the following results.

Lemma 4. Let $A$ be an IFL of $L$ and $B, C \in \operatorname{IFI}\left(A^{*}\right)$. Then

(1) $B \subseteq B+C$ and $C \subseteq B+C$.

(2) $B / C=B / B+C$.

(3) $B+C / B=A$ and $B+C / B \cap C=A$. 
Proof. (1) We have

$$
\begin{aligned}
\mu_{B+C}(x) & =\bigvee_{x=y \vee z}\left(\mu_{B}(y) \wedge \mu_{C}(z)\right) \\
& \geq \mu_{B}(x) \wedge \mu_{C}(0) \quad \text { as } x=x \vee 0 \\
& =\mu_{B}(x) \wedge \mu_{B}(0) \quad \text { since } \mu_{B}(0)=\mu_{C}(0) \\
& =\mu_{B}(x) \quad \text { since } \mu_{B}(0) \geq \mu_{B}(x), \\
v_{B+C}(x) & =\bigwedge_{x=y \vee z}\left(v_{B}(y) \vee v_{C}(z)\right) \\
& \leq v_{B}(x) \vee v_{C}(0) \quad \text { as } x=x \vee 0 \\
& =v_{B}(x) \vee v_{B}(0) \quad \text { since } v_{B}(0)=v_{C}(0) \\
& =v_{B}(x) \quad \text { since } \nu_{B}(0) \leq \nu_{B}(x) .
\end{aligned}
$$

So $B \subseteq B+C$. Similarly $\subseteq B+C$.

(2) We have $B+C \triangleleft A$ (by Theorem 3) and $C \subseteq B+C$ (by (1)). So by Theorem 6 (1),

$$
B / B+C \subseteq B / C
$$

Write $\eta=\{E / E \triangleleft A$ and $E C \subseteq B\}$ and $\xi=\{E / E \triangleleft$ $A$ and $E(B+C) \subseteq B\}$.

Let $E \in \eta$, then $E \triangleleft A$ and $E \subseteq A$. So $E B \subseteq A B$ (by Lemma 1). But $A B \subseteq B$, since $B \triangleleft A$. Hence $E B \subseteq B$ and also $E C \subseteq B$. So By Lemma $1, E(B+C) \subseteq E B+E C \subseteq B+B=B$. Therefore $E \in \xi$. So $\eta \subseteq \xi$. Thus

$$
B / C=\bigcup_{E \in \eta} E \subseteq \bigcup_{E \in \xi} E=B / B+C
$$

From (50) and (51) $B / C=B / B+C$.

(3) We have $B+C \triangleleft A$ and $B \subseteq B+C$. So by Theorem 6 (2), $B+C / B=A$.

Also we have $B \cap C \triangleleft A$ and $B \cap C \subseteq B+C$. Hence by Theorem 6 (2) $B+C / B \cap C=A$.

Theorem 8. Let $A$ be an IFL of $L$ and $\{B i\} i=1,2 \ldots m \in I F I$ $\left(A^{*}\right)$ and $C$ any IFI of $A$. Then $C / \sum_{i=1}^{m} B_{i}=\bigcap_{i=1}^{m}\left(C / B_{i}\right)$.

Proof. We have $B_{1}+B_{2} \triangleleft A$ and $B_{1} \subseteq B_{1}+B_{2}, B_{2} \subseteq B_{1}+B_{2}$. So by Theorem 6 (1), $C / B_{1}+B_{2} \subseteq C / B_{1}$ and $C / B_{1}+B_{2} \subseteq$ $\mathrm{C} / \mathrm{B}_{2}$.

Therefore

$$
C / B_{1}+B_{2} \subseteq C / B_{1} \bigcap C / B_{2} .
$$

Let

$$
\begin{aligned}
& \eta_{1}=\left\{E / E \triangleleft A, E B_{1} \subseteq C\right\}, \\
& \eta_{2}=\left\{E / E \triangleleft A, E B_{2} \subseteq C\right\}, \\
& \eta_{3}=\left\{E / E \triangleleft A, E\left(B_{1}+B_{2}\right) \subseteq C\right\} .
\end{aligned}
$$

Then $\forall x \in L$

$$
\begin{aligned}
\mu_{C / B_{1} \cap C / B_{2}}(x) & =\mu_{C / B_{1}}(x) \wedge \mu_{C / B_{2}}(x) \\
& =\left(\bigvee_{E \in \eta_{1}} \mu_{E}(x)\right) \wedge\left(\bigvee_{E^{\prime} \in \eta_{2}} \mu_{E^{\prime}}(x)\right) \\
& =\bigvee\left\{\left[\mu_{E}(x) \wedge \mu_{E^{\prime}}(x)\right] / E \in \eta_{1}, E^{\prime} \in \eta_{2}\right\} .
\end{aligned}
$$

Similarly

$$
\begin{aligned}
\nu_{C / B_{1} \cap C / B_{2}}(x) & =v_{C / B_{1}}(x) \vee v_{C / B_{2}}(x) \\
& =\left(\bigwedge_{E \in \eta_{1}} \nu_{E}(x)\right) \vee\left(\bigwedge_{E^{\prime} \in \eta_{2}} \nu_{E^{\prime}}(x)\right) \\
& =\wedge\left\{\left[\nu_{E}(x) \vee v_{E^{\prime}}(x)\right] / E \in \eta_{1}, E^{\prime} \in \eta_{2}\right\} .
\end{aligned}
$$

Now let $E \in \eta_{1}$ and $E^{\prime} \in \eta_{2}$. Then $E B_{1} \subseteq C$ and $E^{\prime} B_{2} \subseteq C$. Also $E \cap E^{\prime}$ IFI of $A$, so that

$$
\begin{aligned}
\left(\mathrm{E} \cap E^{\prime}\right)\left(\mathrm{B}_{1}+\mathrm{B}_{2}\right) \subseteq & \left(\mathrm{E} \cap E^{\prime}\right) \mathrm{B}_{1}+\left(\mathrm{E} \cap E^{\prime}\right) \mathrm{B}_{2} \subseteq \mathrm{EB}_{1} \\
& +E^{\prime} \mathrm{B}_{2} \subseteq \mathrm{C}+\mathrm{C}=\mathrm{C} .
\end{aligned}
$$

So $E \cap E^{\prime} \in \eta_{3}$. Hence $\eta_{1} \cap \eta_{2} \subseteq \eta_{3}$. Thus $C / B_{1}+B_{2}=$ $\bigcup_{E \in \eta_{3}} E \supseteq \bigcup_{E \in \eta_{1}, E^{\prime} \in \eta_{2}}\left(E \cap E^{\prime}\right)$.

So

$$
\begin{aligned}
\mu_{C / B_{1}+B_{2}}(x) & \geq \vee \mu_{E \cap E^{\prime}}(x) \\
& =\vee\left[\mu_{E}(x) \wedge \mu_{E^{\prime}}(x)\right] \\
& =\mu_{C / B_{1} \cap C / B_{2}}(x) \text { from (a.3), } \\
\nu_{C / B_{1}+B_{2}}(x) & \leq \wedge v_{E \cap E^{\prime}}(x) \\
& =\wedge\left[\nu_{E}(x) \vee v_{E^{\prime}}(x)\right] \\
& =v_{C / B_{1} \cap C / B_{2}}(x) \text { from (b.3). }
\end{aligned}
$$

Therefore

$$
C / B_{1}+B_{2} \supseteq C / B_{1} \bigcap C / B_{2} .
$$

From (52) and (56) $C / B_{1}+B_{2}=C / B_{1} \cap C / B 2$, hence the result.

\section{References}

[1] K. T. Atanassov, "Intutionistic fuzzy sets," Fuzzy Sets and Systems, vol. 20, no. 1, pp. 87-96, 1986.

[2] K. T. Atanassov, "New operations defined over the intuitionistic fuzzy sets," Fuzzy Sets and Systems, vol. 61, no. 2, pp. 137142,1994

[3] K. H. Kim, "On intuitionistic Q-fuzzy semiprime ideals in semigroups," Advances in Fuzzy Mathematics, vol. 1, no. 1, pp. 15-21, 2006.

[4] K. H. Kim and Y. B. Jun, "Intuitionistic fuzzy interior ideals of semigroups," International Journal of Mathematics and Mathematical Sciences, vol. 27, no. 5, pp. 261-267, 2001. 
[5] K. H. Kim and J. G. Lee, "On intuitionistic fuzzy Bi-ideals of semigroups," Turkish Journal of Mathematics, vol. 29, pp. 201210, 2005.

[6] L. Torkzadeh and M. M. Zahedi, "Intuitionistic fuzzy commutative hyper K-ideals," Journal of Applied Mathematics and Computing, vol. 21, no. 1-2, pp. 451-467, 2006.

[7] M. Akram and W. Dudek, "Interval-valued intuitionistic fuzzy Lie ideals of Lie algebras," World Applied Sciences Journal, vol. 7, no. 7, pp. 812-819, 2009.

[8] K. Hur, S. Y. Jang, and H. W. Kang, "Intuitionistic fuzzy ideals of a ring," Journal of the Korea Society of Mathematical Education. Series B, vol. 12, no. 3, pp. 193-209, 2005.

[9] J. N. Mordeson and D. S. Malik, Fuzzy Commutative Algebra, World Scientific, Singapore, 1998.

[10] A. S. Prajapati, "Residual of ideals of an L-ring," Iranian Journal of Fuzzy Systems, vol. 4, no. 2, pp. 69-82, 2007.

[11] K. V. Thomas and L. S. Nair, "Intuitionistic fuzzy sublattices and ideals," Fuzzy Information and Engineering. In press.

[12] L. Atanassov, "On intuitionistic fuzzy versions of L. Zadeh's extension principle," Notes on Intuitionistic Fuzzy Sets, vol. 13, no. 3, pp. 33-36, 2006.

[13] K. V. Thomas and L. S. Nair, "Operations on intuitionistic fuzzy ideals of a lattice," International Journal of Fuzzy Mathematics. In press. 

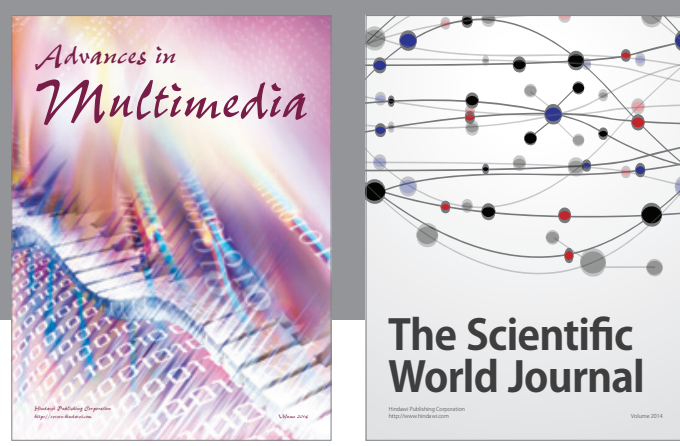

The Scientific World Journal
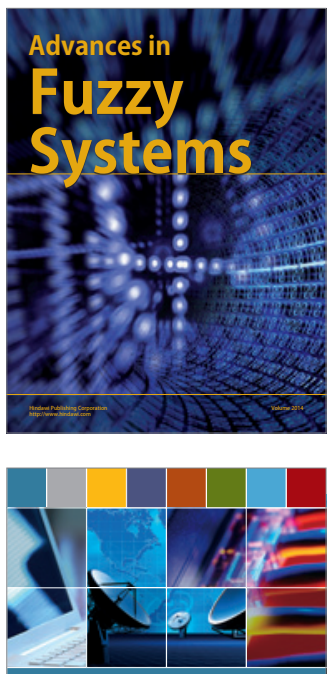

Computer Networks and Communications
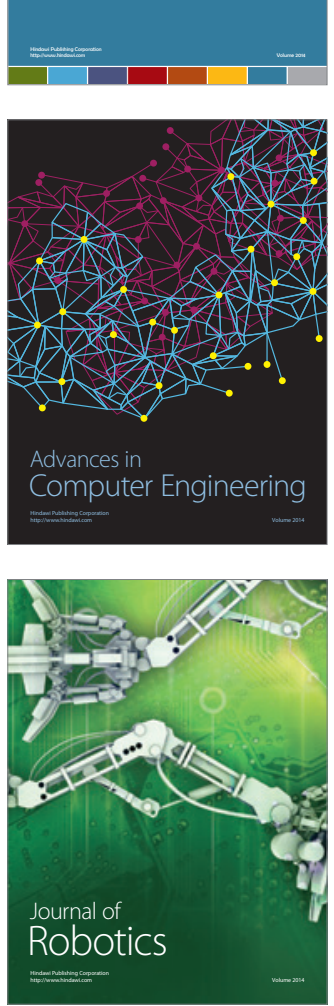
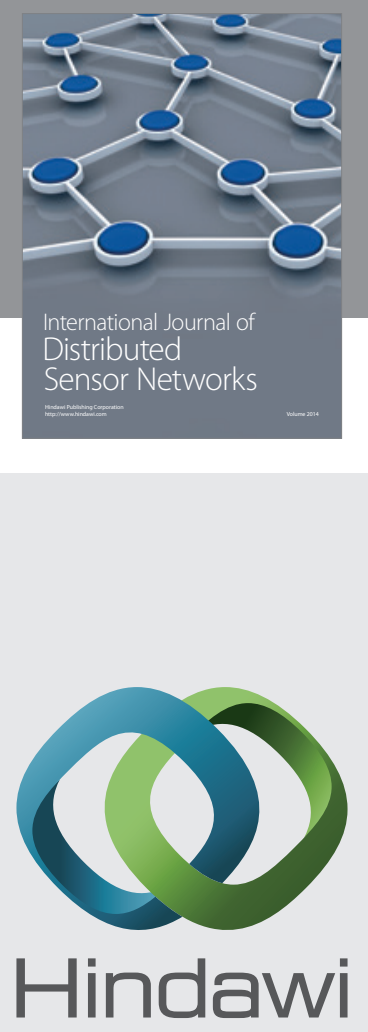

Submit your manuscripts at

http://www.hindawi.com
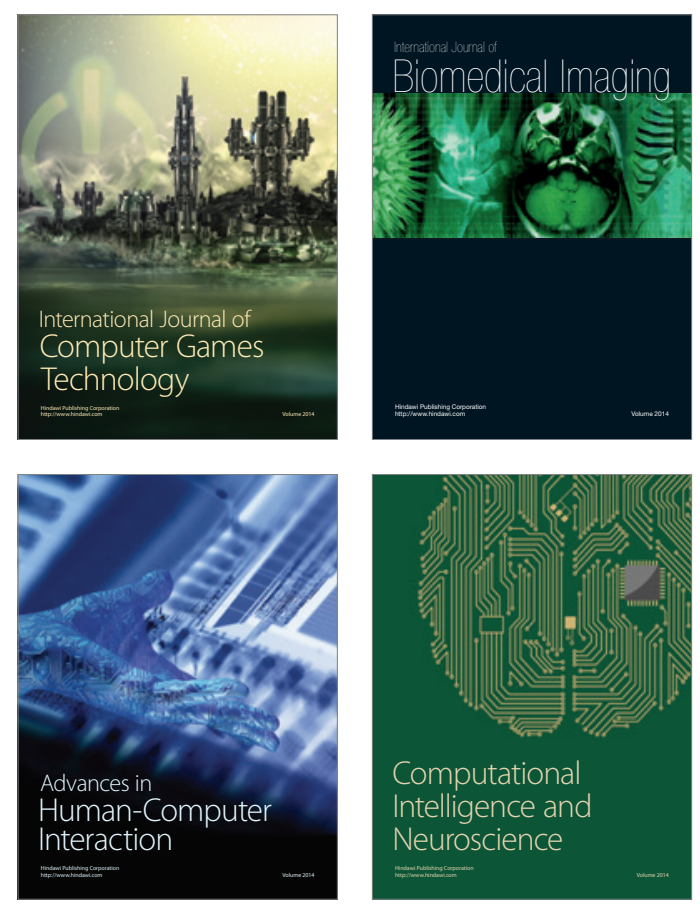
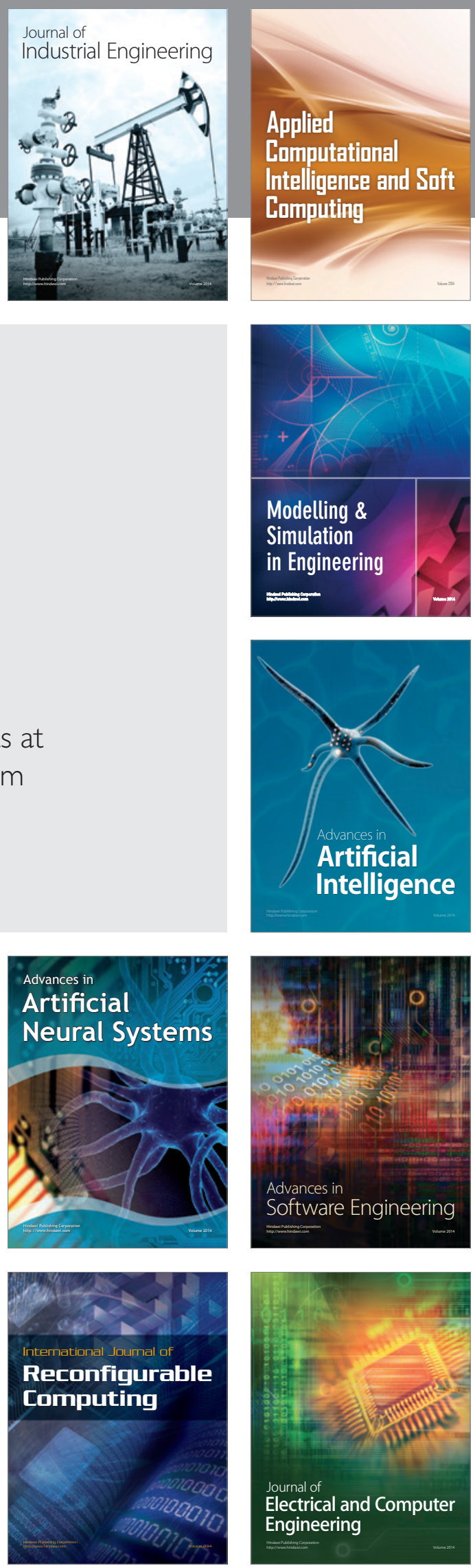